\title{
Physicochemical properties and sensory attributes of local snacks fortified with powdered fish processing by-products and an underutilised fish species
}

\author{
L. ABBEY, M. GLOVER-AMENGOR*, L. HAGAN \& F. P. MBOOM \\ (L.A, M.G-A, L.H \& F.P.M.: CSIR-Food Research Institute, P.O. Box M 20, Accra) \\ *Corresponding author's email: mayamen11@yahoo.com
}

\begin{abstract}
Fish processing by-products and small pelagic fish could provide minerals and protein in diets of vulnerable populations as these could be more affordable than seafood. The study objectives were to determine the proximate nutrient content of tuna processing by-products and burrito fish and also assess the acceptability of fish powder-fortified local carbohydrate snacks. Tuna trimmings, gills, frames and burrito were dried at $55^{\circ} \mathrm{C}$ for eight hours to moisture levels of $4.8 \%$ (trimmings), $8.9 \%$ (frames), $6.8 \%$ (gills) and 6.9\% (burrito). The products were milled and incorporated at varying levels into four local snacks namely: mpotompoto, yakayake, abolo and yam balls. Proximate nutrient levels of both fortified and non-fortified snacks were determined by Official Methods of Analysis (AOAC). Protein contents of mpotompoto-fortified fish products ranged from $3.75 \%$ to $8.5 \%$. Ash also ranged from $1.12 \%$ to $5.54 \%$. The control contained $1.17 \%$. Acceptability tests were conducted on the fortified snacks using a 5-point hedonic scale. On the whole, fortified products showed significantly higher levels of protein and ash over the non-fortified snacks. Acceptance of the snacks by pupils (11-12) years was high enough to warrant incorporation of the fish powders into a school lunch menu.
\end{abstract}

Keywords: Tuna; gills; trimmings; frames; local snacks; fortification

Original scientific paper. Received 12 Dec 2018; revised 15 Aug 2019

\section{Introduction}

Inadequate food supply in terms of meeting energy requirements affects at least 925 million people worldwide (FAO, 2010). Poor rural and urban areas where limited economic resources prevent diversity in diets result in the prevalence of micronutrient deficiency diseases. Fish products are considered as a good source of many micro-minerals, most of which are found in high amounts in fish bones (Abbey et al, 2017; Gordon \& OwusuAdjei, 2011; Ashitey \& Flake, 2010). However, apart from eating small-sized fish species with bones inclusive, bones of larger fish are rarely consumed. Increased use of seafood, including bones, could contribute significantly to reducing micro-mineral and protein malnutrition (Toppe, 2014). Many vulnerable groups, however, cannot afford to buy seafood, particularly in areas where seafood is scarce.

Small pelagic fish which are among the most affordable and healthy fish (Toppe, 2014), could be promoted to solve the economic and logistic challenges in making seafood

Ghana Jnl Agric. Sci. 54 (2), 26 - 35

GJAS is an Open Access Journal and distributed under the terms of the Creative Commons (CC) License [CC BY 4.0] 
accessible and affordable to vulnerable groups. By-products from fish processing represent in many cases more than $50 \%$ of the fish being processed. These by-products are of high nutritional value, and yet are in many cases low-cost products (Toppe, 2014; Kabahenda et al., 2011), so could serve as good dietary supplements for vulnerable groups. The products should, however, be accepted by the target population, and should be introduced into local diets with relative ease. This study is part of an FAO/CSIR-FRI collaborative project, 2015 that aimed at developing lowcost nutrient-dense fish products for National School Feeding Initiatives using low value (underutilized) fish and edible fish by-products. The first part of the study assessed the nutrient content of the fish powders (Abbey et al., 2017). The current study sought to:

- Determine the physicochemical properties of powdered fish-fortified local snacks

- Test the acceptability of powdered fishfortified local snacks by school pupils on the Ghana National School Feeding Programme.

\section{Materials and Methods}

Production of tuna by-products (trimmings, gills and frames) and burrito powder

Tuna trimmings, gills and frames were dried with a Council for Scientific and Industrial Research-Food Research Institute (CSIR-FRI) gas-fuelled dryer for $8 \mathrm{hr}$ to moisture levels of $4.8 \%$ (trimmings), $8.9 \%$ (frames), $6.8 \%$ (gills) and $6.9 \%$ (burrito). The dried fish products were milled with a $250 \mu \mathrm{m}$ sieve hammer mill (Model 160 B; Jacobson, Machinery works, Minneapolis). The fish powders were packaged into polypropylene bags and stored at $-18^{\circ} \mathrm{C}$ until use. The detailed procedure for the preparation of the powdered fish products is described elsewhere (Abbey et al., 2017).

Fortification of carbohydrate foods with tuna by-products and burrito powders

Four local, low nutritional value snacks mpotompoto (yam porridge with added palm oil), yakayake (steamed cassava grits), abolo (baked dehulled and slightly fermented maize paste) and yam balls (boiled, mashed yam made into balls and fried) were prepared in the test kitchen of CSIR-FRI. The snacks were then fortified with the fish powders at percentages of $5,8.8,12.5$, and 16.3 to determine the acceptable levels of inclusion in the products through sensory evaluation. The powdered fish products were added and homogenized as described by Abbey et al. (2015) in the snacks before cooking. Also, each snack had a control with no inclusion of any powder thus having five samples each. The snacks were then assessed in-house for the sensory attributes of appearance, colour, aroma, taste, texture, mouthfeel and overall acceptability on a 9-point hedonic scale by ten semi-trained adult panelists in the sensory laboratory at CSIR-FRI. Following results from the in-house sensory evaluation, three well-accepted products were further developed for the school acceptability testing. These were yakayake, abolo and mpotompoto. Inclusion levels for the various fish products were based on the outcome of the in-house sensory results. 
TABLE 1

Percentage levels of fish powders inclusion in three food products

\begin{tabular}{lclll}
\hline Food Item & Burito & Trimmings & Frames & Gills \\
\hline Yakayake & 8.8 & 8.8 & 8.8 & 12.5 \\
Abolo & 5.0 & 5.0 & 5.0 & 5.0 \\
Mpotompoto & 12.5 & 12.5 & 8.8 & 12.5 \\
\hline
\end{tabular}

School Acceptability Test

The study was carried out in the Greater Accra region of Ghana in one of the coastal communities

\section{Sampling Method/Procedure}

The school for the study was purposively selected in the La Municipality because it was participating in the Ghana School Feeding Programme (GSFP). Class registers were pooled together from class five and six to obtain a sampling frame. All eligible children (ages 11-12 years), present in school on the day of testing and whose caregivers had given assent to their participation $(\mathrm{N}=116)$ were enrolled. There were four groups of fish products so children were made to pick Group 1, 2, 3 or 4 to fall into one of these groups. The children were asked to assess the following attributes of the products: appearance, colour, taste, aroma, texture, mouthfeel and overall acceptability. They were requested to give numerical values to each sensory characteristic assessed using the five 5-point hedonic scale (5-1) with 5 meaning like extremely and 1 meaning dislike extremely. The mean scores for the sensory characteristics of the dishes and the overall acceptance were calculated. The children independently assessed the dishes after the research team explained the terminology to them in English and also the local languages.
Hot food was transported in food warmers to the school just before lunchtime, so hot food was served to the children. A sample was served at a time and each pupil received about $3 \mathrm{~g}$ of food. Ethical clearance for the study was obtained from the Institutional Review Board (IRB) of the Council for Scientific and Industrial Research (CSIR). Parents willing to allow their children to participate in the study either signed or thumb-printed an assent form after the objectives and benefits of the study were explained to them.

\section{Physicochemical analysis of products}

The proximate composition of all fish fortified products and the non-fortified products were determined by standard methods. Moisture content was determined by AOAC 925.10 (AOAC, 2000). Water activity and $\mathrm{pH}$ were determined using the Hygrolab water activity meter and bench type $\mathrm{pH}$ meter respectively. Ash was determined by AOAC 923.03 (AOAC, 2000). Fat was determined by AOAC $923.39 \mathrm{C}$ (AOAC, 2000). Energy was determined by atwater factor. Protein was determined by Kjedahl method (Anon, 1983). Tests were run in duplicates.

\section{Statistical Analysis}

Statistical analysis was done with Statistical Package for Social Scientists (SPSS) version 16. (SPSS, 2005). Analysis of variance (ANOVA) was used to compare the fortified dishes (burrito, frames, gills and trimmings) with the controls.

\section{Results and Discussions}

Physicochemical properties of fish powderfortified snacks

Physicochemical properties of the food products are shown in Tables 2, 3 and 4. The protein contents of mpotompoto fortified with 
Physicochemical properties and sensory attributes of local snacks...

12.5\% burrito, 8.8\% tuna frames, $12.5 \%$ tuna Energy contents ranged from 104.09\% for gills and $12.5 \%$ tuna trimmings were $7.59 \%, \quad 8.8 \%$ inclusion of tuna frames to $149.04 \%$ for $3.75 \%, 6.12 \%$ and $8.5 \%$ respectively. Ash $12.5 \%$ inclusion of tuna gills. The control had contents also ranged from $1.12 \%$ for $12.5 \%$ trimmings to $5.54 \%$ for $8.8 \%$ tuna frames. The control (non-fortified) contained 1.17\%. an energy content of $86.45 \%$. Most inclusions for abolo and yakayake also showed high values of nutrients.

TABLE 2

Proximate results of Mpotompoto with various levels of fish powders inclusion based on the sensory evaluation

\begin{tabular}{llllll}
\hline Parameter & Control & 12.5(\%) Burrito & $8.8(\%)$ Frames & $12.5(\%)$ Gills & 12.5 (\%) Trimmings \\
\hline Moisture g/100 g & 81.51 & $* 69.22$ & $* 73.33$ & $* 67.45$ & $* 70.95$ \\
Ash g/100 g & 1.17 & $* 2.34$ & $* 5.84$ & $* 4.67$ & 1.12 \\
Fat g/100 g & 3.45 & $* 6.4$ & $* 4.15$ & $* 7.5$ & $* 6.02$ \\
Protein g/100g & 1.1 & $* 7.49$ & $* 3.75$ & $* 6.12$ & $* 8.5$ \\
Carbohydrate g/100 g & 12.78 & $* 14.56$ & 12.94 & $* 14.27$ & 13.41 \\
Energy Kcal/100 g & 86.45 & $* 146.28$ & $* 104.09$ & $* 149.04$ & $* 141.82$ \\
\hline
\end{tabular}

Values with asterisk are significantly higher at $5 \%$ level of probability than the control.

TABLE 3

Results of Abolo with inclusion of the various fish powders based on the sensory evaluation

\begin{tabular}{|c|c|c|c|c|c|}
\hline Parameter & Control & $5(\%)$ Burrito & $5(\%)$ Frames & $5(\%)$ & $5(\%)$ Trimmings \\
\hline Moisture (g/100g) & 61.99 & $* 48.76$ & $* 44.24$ & $* 46.44$ & *54.02 \\
\hline Ash (g/100g) & 0.76 & $* 2.77$ & $* 4.34$ & $* 3.21$ & $* 0.83$ \\
\hline Fat $(\mathrm{g} / 100 \mathrm{~g})$ & 0.35 & $* 0.9$ & $* 0.9$ & $* 0.85$ & 0.5 \\
\hline Protein $(g / 100 g)$ & 4.27 & $* 6.25$ & *6.97 & $* 8.60$ & $* 8.90$ \\
\hline Carbohydrate (g/100g) & 32.63 & $* 41.32$ & $* 43.56$ & $* 40.91$ & $* 35.76$ \\
\hline Energy (Kcal/100g) & 150.75 & *198.38 & $* 210.22$ & $* 205.67$ & *183.14 \\
\hline
\end{tabular}

Values with asterisk are significantly higher at $5 \%$ level of probability than the control 
TABLE 4

Proximate results of Yakayake with various levels of fish powders inclusion based on the sensory evaluation

\begin{tabular}{llllll}
\hline Parameter & Control & $8.8(\%)$ Burrito & $\begin{array}{l}8.8(\%) \\
\text { Frames }\end{array}$ & $12.5(\%)$ Gills & $\begin{array}{l}8.8(\%) \\
\text { Trimmings }\end{array}$ \\
\hline Moisture $(\mathrm{g} / 100 \mathrm{~g})$ & 51.44 & $* 67.305$ & $* 49.62$ & $* 48.915$ & $* 48.19$ \\
Ash $(\mathrm{g} / 100 \mathrm{~g})$ & 1.41 & 1.29 & $* 5.97$ & $* 2.07$ & $* 1.78$ \\
Fat $(\mathrm{g} / 100 \mathrm{~g})$ & 0.025 & 0.15 & $* 0.3$ & $* 0.6$ & $* 0.4$ \\
Protein $(\mathrm{g} / 100 \mathrm{~g})$ & 0.69 & $* 7.05$ & $* 3.13$ & $* 8.79$ & $* 7.30$ \\
Carbohydrate $(\mathrm{g} / 100 \mathrm{~g})$ & 46.45 & $* 24.21$ & $* 40.99$ & $* 39.63$ & $* 42.34$ \\
Energy $(\mathrm{Kcal} / 100 \mathrm{~g})$ & 188.75 & $* 126.37$ & $* 179.16$ & $* 199.08$ & $* 202.14$ \\
\hline
\end{tabular}

Values with asterisk are significantly higher at 5\% level of probability than the control

There were significantly higher levels of food fortification imply that the fish products protein in all the fortified products than the control (Table 2). Similarly, ash values were significantly higher in yakayake for all the tuna by-products. That for burrito, however, was not higher than the control value. With mpotompoto, ash contents were significantly higher, except for tuna trimmings, whilst with abolo, all fish products showed significantly higher values of ash. Energy contents were also higher significantly for all fish powder inclusions (Tables 2-4). These results for foodcould be used to increase protein and mineral contents of these low nutrient foods. Earlier work showed that the fish powders contained high levels of protein and iron (Abbey et al., 2017). Being less expensive than the traditional seafood, they could be accessible to lowincome groups. Additionally, processing is cheap as they could be dried as in the current study or even smoked (Gordon \& OwusuAdjei, 2011) to produce fish powder that could be stored for convenient use. 


\section{Sensory Evaluation}

The results of the in-house sensory evaluation are presented in Tables 5-8

TABLE 5

Mean scores and standard deviations for sensory characteristics and overall acceptability of yakayake fortified with fish powder

\begin{tabular}{|c|c|c|c|c|c|c|c|}
\hline & Quality As & sessed $^{2}$ & & & & & \\
\hline SAMPLE & APPEARANCE & COLOUR & TASTE & AROMA & TEXTURE & MOUTHFEEL & $\begin{array}{l}\text { OVERALL } \\
\text { ACCEPTABILITY }\end{array}$ \\
\hline $5(\%)$ Tuna Frames & $6.73 \pm 1.67 b c^{1}$ & $6.73 \pm 1.39 \mathrm{bc}$ & $6.00 \pm 1.57 \mathrm{bcd}$ & $6.33 \pm 1.54 \mathrm{bcd}$ & $6.60 \pm 1.4 \mathrm{bcd}$ & $6.47 \pm 1.41 \mathrm{bcd}$ & $6.53 \pm 1.46 \mathrm{bcd}$ \\
\hline 8.8(\%) Tuna Frames & $6.80 \pm 1.42 \mathrm{bc}$ & $6.87 \pm 1.4 \mathrm{bcd}$ & $6.20 \pm 1.15 \mathrm{bcd}$ & $6.13 \pm 1.41 \mathrm{bcd}$ & $6.73 \pm 1.10 \mathrm{~cd}$ & $6.33 \pm 1.50 \mathrm{bcd}$ & $6.27 \pm 1.16 \mathrm{abcd}$ \\
\hline $12.5(\%)$ Tuna frames & $6.33 \pm 1.39 \mathrm{abc}$ & $6.60 \pm 1.35 b c$ & $5.93 \pm 1.53 \mathrm{abc}$ & $5.60 \pm 1.59 \mathrm{abc}$ & $6.20 \pm 1.82 \mathrm{abc}$ & $5.73 \pm 1.83 \mathrm{ab}$ & $5.93 \pm 1.71 \mathrm{abc}$ \\
\hline 16.3(\%) Tuna Frames & $6.40 \pm 1.72 b c$ & $6.27 \pm 1.58 \mathrm{bc}$ & $5.27 \pm 1.71 \mathrm{ab}$ & $5.27 \pm 1.58 \mathrm{ab}$ & $6.27 \pm 1.58 \mathrm{abcd}$ & $5.53 \pm 1.81 \mathrm{abc}$ & $5.67 \pm 1.78 \mathrm{abc}$ \\
\hline $5(\%)$ Trimmings & $5.60 \pm 1.59 \mathrm{abc}$ & $5.67 \pm 1.72 \mathrm{abc}$ & $6.27 \pm 1.58 \mathrm{bcd}$ & $6.07 \pm 1.16 \mathrm{bc}$ & $6.53 \pm 1.41 \mathrm{bcd}$ & $5.93 \pm 1.39 \mathrm{abc}$ & $5.93 \pm 1.49 \mathrm{abc}$ \\
\hline $12.5(\%)$ Trimmings & $4.47 \pm 2.17 \mathrm{a}$ & $4.67 \pm 2.16 \mathrm{a}$ & $5.07 \pm 1.59 \mathrm{ab}$ & $4.8 \pm 1.74 \mathrm{a}$ & $5.67 \pm 1.23 \mathrm{abc}$ & $4.87 \pm 1.81 \mathrm{a}$ & $5.27 \pm 1.53 \mathrm{abc}$ \\
\hline 16.3(\%) Timmings & $4.00 \pm 2.20 \mathrm{a}$ & $4.06 \pm 2.12 \mathrm{a}$ & $4.73 \pm 1.71 \mathrm{a}$ & $4.53 \pm 1.81 \mathrm{a}$ & $5.33 \pm 1.68 \mathrm{abbc}$ & $4.60 \pm 1.96 \mathrm{a}$ & $4.73 \pm 1.44 \mathrm{a}$ \\
\hline $8.8(\%)$ Trimmings & $5.33 \pm 1.84 \mathrm{ab}$ & $5.46 \pm 1.88 \mathrm{abc}$ & $6.07 \pm 1.33 \mathrm{bcd}$ & $5.87 \pm 1.18 \mathrm{abc}$ & $6.47 \pm 1.51 \mathrm{bcd}$ & $5.73 \pm 1.58 \mathrm{bcd}$ & $6.07 \pm 1.49 \mathrm{bcd}$ \\
\hline Control & $7.93 \pm 1.03 \mathrm{~cd}$ & $8.07 \pm 1.03 \mathrm{~d}$ & $7.60 \pm 1.18 \mathrm{e}$ & $7.27 \pm 1.28 \mathrm{~cd}$ & $7.47 \pm 1.19 \mathrm{e}$ & $7.47 \pm 0.92 \mathrm{e}$ & $7.73 \pm 0.96 \mathrm{e}$ \\
\hline 12.5(\%) Gills & $6.07 \pm 1.67 \mathrm{abc}$ & $6.07 \pm 1.75 \mathrm{bc}$ & $5.87 \pm 1.60 \mathrm{abc}$ & $5.80 \pm 1.61 \mathrm{abc}$ & $6.33 \pm 1.23 b b c$ & $6.33 \pm 1.23 b c$ & $6.00 \pm 1.41 \mathrm{abc}$ \\
\hline 8.8(\%) Gills & $5.87 \pm 1.68 \mathrm{abc}$ & $6.47 \pm 1.68 \mathrm{bc}$ & $6.00 \pm 1.46 \mathrm{bcd}$ & $5.86 \pm 1.66 \mathrm{abc}$ & $6.47 \pm 1.41 \mathrm{bbc}$ & $5.80 \pm 1.78 \mathrm{bc}$ & $6.07 \pm 1.67 \mathrm{bc}$ \\
\hline $16.3(\%)$ Gills & $5.53 \pm 2.03 \mathrm{ab}$ & $5.13 \pm 1.96 \mathrm{ab}$ & $5.20 \pm 1.86 \mathrm{ab}$ & $5.07 \pm 1.83 \mathrm{abc}$ & $6.07 \pm 1.39$ & $5.00 \pm 1.77$ & $5.40 \pm 1.76$ \\
\hline $5(\%)$ Gills & $7.13 \pm 1.19 b c$ & $7.13 \pm 0.99 \mathrm{~d}$ & $6.67 \pm 0.98 \mathrm{bcd}$ & $6.67 \pm 1.11 \mathrm{bcd}$ & $6.93 \pm 1.16$ & $6.73 \pm 1.22$ & $6.87 \pm 0.92$ \\
\hline $5(\%)$ Burito & $8.07 \pm 0.88 \mathrm{~d}$ & $7.87 \pm 0.83 \mathrm{~cd}$ & $7.67 \pm 1.11 \mathrm{e}$ & $7.40 \pm 1.45 \mathrm{cde}$ & $7.67 \pm 1.05$ & $7.67 \pm 1.05$ & $7.47 \pm 1.64$ \\
\hline $12.5(\%)$ Burito & $6.20 \pm 1.90 \mathrm{abc}$ & $5.87 \pm 1.73 \mathrm{abc}$ & $5.87 \pm 1.96 \mathrm{abc}$ & $5.87 \pm 1.46 \mathrm{abc}$ & $6.40 \pm 1.45$ & $5.53 \pm 1.81$ & $5.67 \pm 1.40$ \\
\hline $8.8(\%)$ Burito & $7.27 \pm 1.22 \mathrm{bc}$ & $7.13 \pm 0.99 \mathrm{~cd}$ & $6.60 \pm 1.35 \mathrm{bcd}$ & $6.13 \pm 1.88 \mathrm{abc}$ & $6.53 \pm 1.92$ & $6.33 \pm 1.50$ & $6.27 \pm 1.98$ \\
\hline $16.3(\%)$ Burito & $5.47 \pm 2.17 \mathrm{a}$ & $5.27 \pm 2.12 \mathrm{abc}$ & $4.87 \pm 1.81 \mathrm{a}$ & $5.13 \pm 1.85 \mathrm{ab}$ & $5.80 \pm 2.18$ & $5.20 \pm 1.90$ & $5.13 \pm 1.97$ \\
\hline $\begin{array}{l}{ }^{1} \text { Means in the s } \\
\text { probability; }{ }^{2} \text { ser } \\
\text { 2-dislike very m } \\
\text { moderately, } 8 \text {-li }\end{array}$ & $\begin{array}{l}\text { ke very much } \\
\text { k-dislik }\end{array}$ & $\begin{array}{l}\text { moderately, } \\
\text { 9-like extre }\end{array}$ & $\begin{array}{l}\text { 4-dislike slig } \\
\text { mely }\end{array}$ & ghtly, 5-neit & r like nor & slike, 6-like & $\begin{array}{l}\text { evel of } \\
\text { e extremely, } \\
\text { lightly, 7-like }\end{array}$ \\
\hline
\end{tabular}


TABLE 6

Mean scores and standard deviations for sensory characteristics and overall acceptability of abolo fortified with fish powder

\begin{tabular}{|c|c|c|c|c|c|c|c|}
\hline \multirow[b]{2}{*}{ SAMPLE } & \multicolumn{2}{|c|}{ Quality Assessed ${ }^{2}$} & \multirow[b]{2}{*}{ TASTE } & \multirow[b]{2}{*}{ AROMA } & \multirow[b]{2}{*}{ TEXTURE } & \multirow[b]{2}{*}{ MOUTHFEEL } & \multirow[b]{2}{*}{$\begin{array}{l}\text { OVERALL } \\
\text { ACCEPTABILITY }\end{array}$} \\
\hline & APPEARANCE & COLOUR & & & & & \\
\hline Control & $7.63 \pm 0.89 \mathrm{e}^{1}$ & $7.56 \pm 0.81 \mathrm{e}$ & $7.63 \pm 0.81 \mathrm{e}$ & $7.56 \pm 0.96 \mathrm{e}$ & $7.19 \pm 1.05 \mathrm{e}$ & $7.44 \pm 0.89 \mathrm{e}$ & $7.38 \pm 0.96 \mathrm{e}$ \\
\hline $\begin{array}{l}8.8(\%) \\
\text { Trimmings }\end{array}$ & $4.75 \pm 1.88 \mathrm{abc}$ & $4.50 \pm 1.86 \mathrm{abc}$ & $4.88 \pm 1.63 \mathrm{abc}$ & $4.94 \pm 1.57 \mathrm{abc}$ & $4.88 \pm 1.78 \mathrm{abc}$ & $4.88 \pm 1.78 \mathrm{abc}$ & $5.06 \pm 1.65 \mathrm{abcd}$ \\
\hline $\begin{array}{l}16.3(\%) \\
\text { Timmings }\end{array}$ & $3.88 \pm 1.86 \mathrm{a}$ & $3.56 \pm 1.96 \mathrm{a}$ & $4.67 \pm 1.54 \mathrm{abc}$ & $4.50 \pm 1.51 \mathrm{abc}$ & $4.00 \pm 1.67 b c$ & $4.53 \pm 1.68 \mathrm{abc}$ & $4.06 \pm 1.48 \mathrm{a}$ \\
\hline 8.8(\%) Gills & $6.19 \pm 1.33$ bcde & $6.00 \pm .1 .26$ bcde & $6.19 \pm 1.60$ bcde & $5.81 \pm 1.42$ bcde & $5.94 \pm 1.44$ bcde & $5.67 \pm 1.39$ bcde & $5.88 \pm 1.20 \mathrm{bcde}$ \\
\hline $\begin{array}{l}5(\%) \\
\text { Trimmings }\end{array}$ & $5.69 \pm 1.45 \mathrm{bcde}$ & $\begin{array}{l}5.50 \pm 1.63 \\
\text { abcde }\end{array}$ & $5.94 \pm 1.53$ abcde & $\begin{array}{l}5.75 \pm 1.39 \\
\text { abcde }\end{array}$ & $6.06 \pm 1.44$ bcde & $6.06 \pm 1.18$ bcde & $6.13 \pm 1.20 \mathrm{cde}$ \\
\hline $5(\%)$ Gills & $6.63 \pm 1.15 \mathrm{cde}$ & $6.69 \pm 1.08 \mathrm{de}$ & $6.81 \pm 1.38 \mathrm{de}$ & $6.50 \pm 1.21 \mathrm{cde}$ & $6.63 \pm 1.54 \mathrm{cde}$ & $6.63 \pm 1.36 \mathrm{cde}$ & $6.63 \pm 1.31 \mathrm{de}$ \\
\hline $\begin{array}{l}12.5(\%) \\
\text { Trimmings }\end{array}$ & $4.25 \pm 1.95 \mathrm{abc}$ & $4.06 \pm 1.91 \mathrm{ab}$ & $4.56 \pm 1.59 \mathrm{abc}$ & $4.69 \pm 1.49 \mathrm{abc}$ & $4.13 \pm 2.13 \mathrm{ab}$ & $4.63 \pm 1.71 \mathrm{abc}$ & $4.38 \pm 1.63 \mathrm{abc}$ \\
\hline $5(\%)$ Burito & $7.47 \pm 0.99 \mathrm{de}$ & $7.40 \pm 1.06 \mathrm{de}$ & $6.87 \pm 1.30 \mathrm{de}$ & $6.53 \pm 1.81 \mathrm{de}$ & $6.80 \pm 1.52 \mathrm{de}$ & $6.67 \pm 1.91 \mathrm{de}$ & $6.87 \pm 1.60 \mathrm{e}$ \\
\hline $\begin{array}{l}16.3(\%) \\
\text { Burito }\end{array}$ & $5.07 \pm 2.02 \mathrm{bcd}$ & $5.20 \pm 2.08 \mathrm{abcd}$ & $4.40 \pm 1.76 \mathrm{abc}$ & $4.40 \pm 1.76 \mathrm{abc}$ & $5.20 \pm 1.78 \mathrm{abcd}$ & $4.07 \pm 1.98 \mathrm{a}$ & $4.33 \pm 1.76 \mathrm{ab}$ \\
\hline $8.8(\%)$ Burito & $6.80 \pm 1.08 \mathrm{de}$ & $6.87 \pm 1.46 \mathrm{de}$ & $5.87 \pm 1.88 \mathrm{de}$ & $5.47 \pm 1.85 \mathrm{de}$ & $6.33 \pm 1.84 \mathrm{de}$ & $5.27 \pm 2.28 \mathrm{de}$ & $5.60 \pm 1.92 \mathrm{abcde}$ \\
\hline $\begin{array}{l}12.5(\%) \\
\text { Burito }\end{array}$ & $5.67 \pm 2.26 \mathrm{bcde}$ & $5.73 \pm 2.21 \mathrm{bcde}$ & $5.40 \pm 2.06 \mathrm{abcd}$ & $4.73 \pm 2.09 \mathrm{ab}$ & $6.00 \pm 1.81 \mathrm{bcd}$ & $4.87 \pm 1.99 \mathrm{abc}$ & $5.00 \pm 2.07 \mathrm{abcd}$ \\
\hline
\end{tabular}

${ }^{1}$ Means in the same column followed by different alphabets are significantly different at $5 \%$ level of probability; ${ }^{2}$ sensory attributes were evaluated on a 9-point hedonic scale as follows: 1- dislike extremely, 2-dislike very much, 3-dislike moderately, 4-dislike slightly, 5-neither like nor dislike, 6-like slightly, 7-like moderately, 8-like very much, 9-like extremely

TABLE 7

Mean scores and standard deviations for sensory characteristics and overall acceptability of yam balls fortified with fish powder

\begin{tabular}{llllllll}
\hline \multicolumn{9}{c}{ Quality Assessed $^{2}$} & & & & \\
SAMPLE & APPEARANCE & COLOUR & TASTE & AROMA & TEXTURE & MOUTHFEEL & $\begin{array}{l}\text { OVERALL } \\
\text { ACCEPTABILITY }\end{array}$ \\
\hline $16.3(\%)$ Burito & $5.13 \pm 1.85 \mathrm{abcd}^{1}$ & $5.13 \pm 1.73 \mathrm{abcd}$ & $5.57 \pm 1.87 \mathrm{abcd}$ & $5.60 \pm 1.80 \mathrm{abcd}$ & $5.80 \pm 1.89 \mathrm{abcde}$ & $5.27 \pm 1.98 \mathrm{abcd}$ & $5.20 \pm 1.78 \mathrm{abcd}$ \\
$12.5(\%)$ Burito & $5.87 \pm 1.68 \mathrm{bcde}$ & $5.67 \pm 1.68 \mathrm{abcd}$ & $5.53 \pm 1.60 \mathrm{abcd}$ & $5.93 \pm 1.83 \mathrm{bcd}$ & $5.80 \pm 1.52 \mathrm{bcd}$ & $5.73 \pm 1.62 \mathrm{bcd}$ & $5.43 \pm 1.50 \mathrm{abc}$ \\
$8.8(\%)$ Burito & $6.60 \pm 1.45 \mathrm{de}$ & $6.45 \pm 1.55 \mathrm{de}$ & $6.73 \pm 1.39 \mathrm{de}$ & $6.80 \pm 1.42 \mathrm{de}$ & $6.27 \pm 1.94 \mathrm{de}$ & $6.67 \pm 1.59 \mathrm{de}$ & $6.67 \pm 1.35 \mathrm{de}$ \\
$5(\%)$ Burito & $7.67 \pm 0.82 \mathrm{e}$ & $7.60 \pm 0.63 \mathrm{e}$ & $7.80 \pm 0.41 \mathrm{e}$ & $7.73 \pm 0.59 \mathrm{e}$ & $7.80 \pm 0.68 \mathrm{e}$ & $7.93 \pm 0.59 \mathrm{e}$ & $8.00 \pm 0.38 \mathrm{e}$ \\
\hline
\end{tabular}


${ }^{1}$ Means in the same column followed by different alphabets are significantly different at $5 \%$ level of probability; ${ }^{2}$ sensory attributes were evaluated on a 9-point hedonic scale as follows: 1- dislike extremely, 2-dislike very much, 3-dislike moderately, 4-dislike slightly, 5-neither like nor dislike, 6-like slightly, 7-like moderately, 8-like very much, 9-like extremely

TABLE 8

Mean scores and standard deviations for sensory characteristics and overall acceptability of mpotompoto fortified with fish powder

\begin{tabular}{llllllll}
\hline SAMPLE & APPEARANCE & COLOUR & TASTE & AROMA & TEXTURE & MOUTHFEEL & ACCEPTABILITY \\
\hline $5(\%)$ Burittoo & $8.00 \pm 0.53 \mathrm{~b}^{1}$ & $8.00 \pm 0.53 \mathrm{~b}$ & $6.93 \pm 1.33 \mathrm{ab}$ & $7.33 \pm 0.90 \mathrm{~b}$ & $7.60 \pm 0.83 \mathrm{~b}$ & $7.47 \pm 1.13 \mathrm{~b}$ & $7.67 \pm 0.72 \mathrm{~b}$ \\
$16.3(\%)$ Burito & $6.60 \pm 0.74 \mathrm{ab}$ & $6.47 \pm 1.13 \mathrm{ab}$ & $5.86 \pm 0.95 \mathrm{a}$ & $6.31 \pm 1.28 \mathrm{ab}$ & $6.07 \pm 1.28 \mathrm{a}$ & $6.27 \pm 0.96 \mathrm{ab}$ & $6.27 \pm 0.88 \mathrm{ab}$ \\
$8.8(\%)$ Burito & $7.47 \pm 0.83 \mathrm{ab}$ & $7.73 \pm 0.59 \mathrm{~b}$ & $7.47 \pm 1.25 \mathrm{~b}$ & $7.47 \pm 1.19 \mathrm{~b}$ & $7.60 \pm 0.91 \mathrm{~b}$ & $7.53 \pm 0.99 \mathrm{~b}$ & $7.47 \pm 0.99 \mathrm{~b}$ \\
$12.5(\%)$ Burito- & $6.33 \pm 1.18 \mathrm{a}$ & $6.27 \pm 1.22 \mathrm{ab}$ & $6.40 \pm 1.55 \mathrm{ab}$ & $6.80 \pm 1.01 \mathrm{ab}$ & $6.80 \pm 1.37 \mathrm{ab}$ & $6.80 \pm 1.15 \mathrm{~b}$ & $6.73 \pm 1.03 \mathrm{ab}$ \\
\hline
\end{tabular}

${ }^{1}$ Means in the same column followed by different alphabets are significantly different at $5 \%$ level of probability; ${ }^{2}$ sensory attributes were evaluated on a 9-point hedonic scale as follows: 1- dislike extremely, 2-dislike very much, 3-dislike moderately, 4-dislike slightly, 5-neither like nor dislike, 6-like slightly, 7-like moderately, 8-like very much, 9-like extremely

\section{Acceptability of products during school} feeding test

Results of the acceptability of the three products for the school feeding trial are presented below.

\section{Abolo}

The sensory profile (Figure 1) indicates that abolo fortified with the powder of tuna frames was rated highly compared to the abolo fortified with other fishes. Panelists liked the taste of all the abolo samples except the abolo fortified with trimmings.

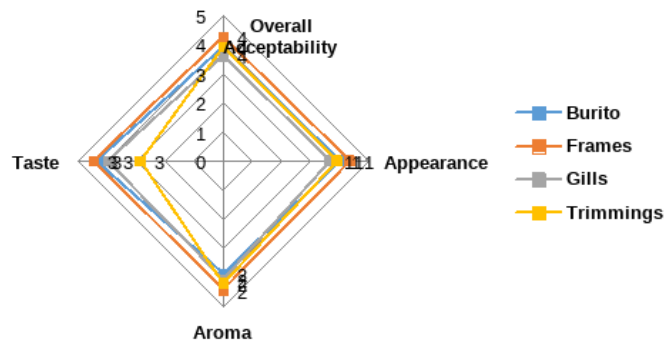

Fig. 1: Sensory profile of Abolo fortified with different varieties of fishes

\section{Yakayake}

Yakayake fortified with the various fishes rated lower in terms of appearance, aroma, and taste. However, trimmings in yakayake was rated high (4) in terms of overall acceptability.

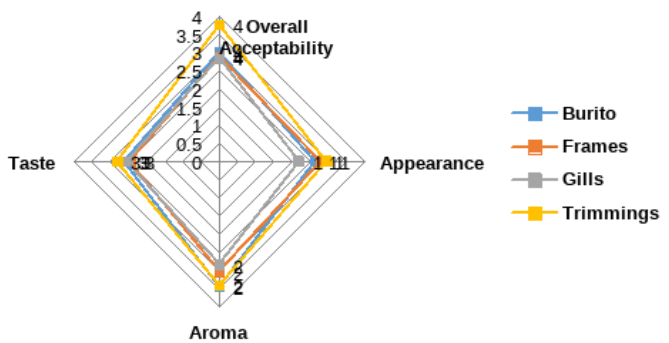

Fig. 2: Sensory profile of Yakayake fortified with different varieties of fish 


\section{Mpotompoto}

The profile in Figure 3 shows that mpotompoto fortified with the various fishes was rated highest in all attributes. A general trend of a somewhat even rating was observed for all the varieties of fish used.

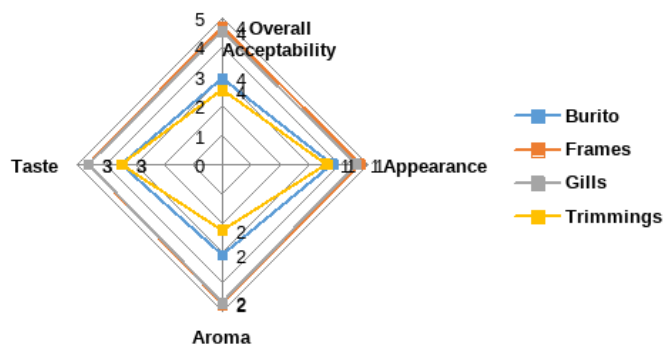

Fig. 3: Sensory profile of Mpotompoto fortified with different varieties of fish

\section{In house Panelists}

The appearance of the food products fortified with the various fishes was liked extremely except the appearance of the food products made from trimmings that were liked slightly according to the hedonic scale used. In exception of the higher concentrations of gills the appearance of the reference samples was not significantly different from all the other samples (Table 5, 6 and 8). The colour of all the samples was liked very much except the colour of food products fortified with trimmings (Table 5, 6, 7 and 8) and was significantly different from the reference sample (Tables 5 and 6). The aromas of all the samples were liked and were not significantly different from each other. On the whole, the taste of the fishes in the food products was acceptable to the panelists as is evident by the fact that the taste was not rated below 5 except the taste of food products with trimmings. The taste for the samples with trimmings was not accepted because of its bitterness. On the other hand, the taste of mpotompoto was rated high (above 7) on the hedonic scale, meaning panelists liked the taste of the fishes in the mpotompoto even with the high concentrations. According to the analysis of variance, there was no significant difference in panelists liking of the taste of samples but there was difference between the samples with trimmings and the reference samples. Consequently, panelists liked the mouthfeel for all the samples but did not like the mouthfeel for the high concentrations of the fishes in the food products. The ratings for these samples were below 5 on the hedonic scale.

\section{Overall Acceptability}

All the products were acceptable as they were rated above 6 (like slightly) and they were not significantly different from the reference samples except food products with trimmings.

\section{School-Based Testing}

Panelists liked the taste of all the abolo samples except the abolo fortified with trimmings. Yakayake fortified with the various fishes rated lower in terms of appearance, aroma, and taste. However, trimmings in yakayake was rated high (4) in terms of overall acceptability. The profile in Figure 3 shows that mpotompoto fortified with the various fishes was rated highest in all attributes. A general trend of a somewhat even rating was observed for all the varieties of fish used. Hence these fish powders have the potential for improving the nutritional status of children on School Feeding Programmes, and would help reduce protein and mineral malnutrition. 


\section{Conclusion}

The fortified foods contained significantly higher levels of protein, ash and energy over the controls, and thus have the potential to improve the nutritional status of low-income populations as they are more accessible and affordable. All the products were accepted by the panelists except the food products with trimmings which were not acceptable because of the bitterness. Consuming small-sized fish species whole, and bones of large fish could, therefore, contribute significantly to reducing the level of mineral and protein malnutrition in vulnerable groups.

\section{Conflict of Interest}

There is no conflict of interest.

\section{Acknowledgement}

The authors wish to thank the Food and Agriculture Organization (FAO) and the Food Research Institute of the Council for Scientific and Industrial Research (CSIR-FRI) for the financial support towards this study.

\section{REFERENCES}

Abbey, L., Glover-Amengor, M., Atikpo, M., Atter, A., Dowuona, S., Mireku. E., Mboom FP., Manu, S., Toppe. J., \& Kiran, M. (2015) Report on Development of LowCost Nutrient Dense Fish Products Based on Low-Value Fish and Fish Byproducts using small and medium scale Processing and Preservation methods that Stabilize the Nutritional Value and ensure the safety of the products. Submitted to the FAO, Rome. CSIR-FRI/CR/ALD/2015/002.

Abbey, L., Glover-Amengor, M., Atikpo, M. O., Atter, A. \& Toppe J. (2017) Nutrient content of fish powder from low-value fish and fish byproducts. Food Science and Nutrition, 5 (3), $374-379$.
Anon. (1983) Determination of Kjeldahl Protein in fish and fish products using the Kjeltec Auto System and $\mathrm{Se}$ or $\mathrm{Hg}$ Catalyst. Application Note. ASN 56, $1-2$.

Ashitey, E. \& Flake, L. (2010) Exporter guide. Global Aquaculture Information Network. GAIN Report No: GH1002. pp. 16.

FAO (2010) The state of food insecurity in the world 2010: eradicating world hunger key to achieving the Millennium Development goals. Rome.

Gordon, A., Pulis, A. \& Owusu-Adjei, E. (2011) Smoked marine fish from Western Region, Ghana: A value chain assessment. WorldFish Center. USAID Integrated Coastal and Fisheries Government.

Kabahenda, M. K, Amega, R., Okalany, E., Husken, S. M. C. \& Heck, S. (2011) Protein and Micronutrient Composition of LowValue Fish Products Commonly Marketed in the Lake Victoria Region. World Journal of Agricultural Sciences 7 (5), 521 - 526.

Official Methods of Analysis. (2000a) Association of official analytical chemists. AOAC 925.10 17 th ed. AOAC International, Washington DC, USA.

Official Methods of Analysis. (2000b) Association of official analytical chemists. AOAC 923.03 17 th ed. AOAC International, Washington DC, USA.

Official Methods of Analysis. (2000c) Association of official analytical chemists. AOAC 923.39C 17th ed. AOAC International, Washington DC, USA.

SPSS 16 for Windows. (2005) SPSS 16 for windows. Illinois, Chicago.

Toppe, J. (2014) The nutritional benefits of fish are unique. EUROFISH Magazine, Issue 3. 